MATHEMATICS OF COMPUTATION

Volume 67, Number 222, April 1998, Pages 759-772

S 0025-5718(98)00958-2

\title{
EXCEPTIONAL UNITS IN A FAMILY OF QUARTIC NUMBER FIELDS
}

\author{
G. NIKLASCH AND N.P. SMART
}

\begin{abstract}
We determine all exceptional units among the elements of certain groups of units in quartic number fields. These groups arise from a oneparameter family of polynomials with two real roots.
\end{abstract}

\section{INTRODUCTION}

There has been much interest in exceptional units over recent years - partly for their own sake, see for instance [2], [5], and [6], and partly because they arise when solving diophantine equations of various classical types. For instance 2-term unit equations arise in the algorithm of Tzanakis and de Weger for solving Thue and Thue-Mahler equations, see [15] and [16], and in the work of Smart on solving discriminant form equations, [13]. In recent years a number of authors have considered solving parametrized families of diophantine equations, see [14], [7], [11] and [8]. For any given number field the algorithm in [12] can be used in principle to determine all the exceptional units within the field. Treating a parametric family of fields in this way requires a method for controlling the fundamental units; however, it is quite feasible to investigate exceptional units in a parametrized family of equation orders, as we will show.

Up to isomorphism, only finitely many number fields of unit rank zero or one contain exceptional units which do not come from a proper subfield, and Nagell determined all of these in a series of papers in the late 1960s, [9]. In [10] the first author suggested an approach for finding all exceptional units in parametrized families of number fields of unit rank at least two. Here we shall discuss one such family in detail, intending to provide an example on which the investigation of other families can be modeled, as well as to illustrate the power of recent estimates for linear forms in logarithms.

Recall that a unit $\varepsilon$ of a commutative ring with 1 is exceptional if $1-\varepsilon$ is also a unit; in other words, if there exists a unit $\varepsilon^{\prime}$ such that

$$
\varepsilon+\varepsilon^{\prime}=1 \text {. }
$$

In our case, the underlying rings will be monogenic subrings $R=\mathbb{Z}[\theta]$ of the rings of integers $\mathcal{O}_{K}$ of certain number fields $K=\mathbb{Q}(\theta)$, and the units $\varepsilon$ will be taken from an explicitly presented subgroup of the group of units $R^{*}$, whereas $\varepsilon^{\prime}$ will a priori live in $R^{*}$. We can test whether $\varepsilon$ is exceptional by checking whether the absolute

Received by the editor October 18, 1996.

1991 Mathematics Subject Classification. Primary 11D61, 11R27, 11J86, 11J25.

Key words and phrases. Exceptional units, Baker's method, diophantine approximation.

(C)1998 American Mathematical Society 
norm $\left|N_{K / \mathbb{Q}}(\varepsilon-1)\right|$ equals 1 , norms always being taken from $K$ to $\mathbb{Q}$. Specifically, consider the family of polynomials

$$
f_{a}(x)=x^{4}+a x^{3}+x^{2}+a x-1
$$

where $a \in \mathbb{Z}_{>0}$. Note that these polynomials are invariant under the joint substitution $(x, a) \mapsto(-x,-a)$, so it is no loss of generality to exclude the values $a<0$, and that they are irreducible because their reductions modulo 2 and 3 are incompatible with linear and with quadratic factors, respectively. The discriminant of $f_{a}$ is

$$
-4 a^{6}-47 a^{4}-112 a^{2}-400,
$$

hence always negative. Thus each $f_{a}$ has two real roots and a pair of complex roots. Let $\theta$ denote any root of $f_{a}$ in abstracto and let $R, K$ be as above, the dependence on $a$ being understood. The full unit group $\mathcal{O}_{K}^{*}$ is the direct product of $\{ \pm 1\}$ with a free abelian group of rank 2 .

As explained in [10, section 4.5], our family is one of several for which $\eta_{1}=\theta^{-1}$ and $\eta_{2}=\theta^{2}+1$ are always units of $R$. Thus we have, for each $a \geq 1$, the trivial exceptional units, namely

$$
\theta^{2}+1, \quad-\theta^{2}, \quad \theta^{2} /\left(1+\theta^{2}\right) \text { and their inverses. }
$$

Recall, e. g. from [6, section 2], that the group $\mathcal{H}$ of order 6 of fractional linear transformations generated by $\omega \mapsto 1 / \omega$ and $\omega \mapsto 1-\omega$ acts on the exceptional units of any ring. Let $G$ be the subgroup of units of $\mathcal{O}_{K}^{*}$ generated by $\left\{-1, \eta_{1}, \eta_{2}\right\}$. We shall see shortly that it is of finite index in $\mathcal{O}_{K}^{*}$ when $a \geq 1$. (This is not true when $a=0$, and this is one reason why we have excluded that case; it will be treated on another occasion in the context of quartic fields of mixed signature with a quadratic subfield.)

Our principal result is:

Theorem. For $a \geq 2$, there are no nontrivial exceptional units in $G$. For $a=1$, there are only two nontrivial $\mathcal{H}$-orbits of exceptional units, represented by $\eta_{1}^{-1}+1=$ $\eta_{1} \eta_{2}^{-1}$ and by $\eta_{1}^{-1} \eta_{2}^{3}$.

Remark. For $a=1$, and for many other values of $a$ including all $a<4000$, we have checked that $G=\mathcal{O}_{K}^{*}$. A number-geometric argument will show that at any rate $G=R^{*}$, essentially because a nontrivial coset of $G$ in $R^{*}$ would contain elements whose absolute discriminants are smaller than $4 a^{6}$. This argument depends on $K$ not having a quadratic subfield. We omit the details since we will not use this anywhere.

Outline of this paper. After a short preparatory section exploiting the action of $\mathcal{H}$, our first substantial task will be to determine small intervals containing the logarithms of the generating units (more precisely, of the absolute values of their real and complex embeddings). This is easier when $a$ is large, and we will often treat the smallest values $1 \leq a \leq 5$ separately. In section 4 , we prove two inequalities involving the exponents $b_{1}, b_{2}$ of $\theta=\eta_{1}^{-1}$ and $\eta_{2}$ occurring in a putative nontrivial exceptional unit from $G$. The "gap inequality" says that the exponents must be of very disparate sizes and thus, since they are integers and (as we shall see) neither of them is zero, one of them must be very large. This implies that our candidate must have an embedding extremely close to 1, the "diophantine approximation inequality". Using the latter, we can then replace the crude first version of the gap inequality with a sharp one. In section 5 , we confront our inequalities with a recent 
lower bound on linear forms in two logarithms from [4], obtaining explicit upper bounds on $a$ and on the exponents $b_{j}$. Section 6 is devoted to describing the (small) amount of machine computation needed to exclude any nontrivial solutions in the remaining range. The whole approach is based on ideas introduced by Thomas [14] and refined by Mignotte [7].

Computer-aided formula manipulations and computations were performed by the second author using LiDIA on a Silicon Graphics R5000 Workstation and, independently, by the first author using PARI/GP 1.39.13 on an IntelP100 system under LiNuX.

\section{Preparatory steps}

We keep the notations introduced above, and fix embeddings of $K$ into $\mathbb{C}$ representing the real and complex places as follows: The first sends $\theta$ to the real root of $f_{a}$ between 0 and 1 , the second to the real root between $-a-1$ and $-a$, and the third to the complex root of positive imaginary part. For elements $\alpha \in K$ and $k \in\{1,2,3\}$, let $\alpha^{(k)}$ denote the image of $\alpha$ under the $k$-th embedding.

Suppose that $G$ contains a nontrivial exceptional unit $\omega$. Since we are free to replace $\omega$ by one of its images under $\mathcal{H}$, we may assume that $\left.\omega^{(1)} \in\right] 1,2[$. Since $\eta_{1}^{(1)}$ and $\eta_{2}^{(1)}$ are positive, we have the ansatz

$$
\omega=\theta^{b_{1}} \eta_{2}^{b_{2}}=\eta_{1}^{-b_{1}} \eta_{2}^{b_{2}}
$$

with unknown integer exponents $b_{1}, b_{2}$ distinct from $(0,0)$ and $(0,1)$.

The importance of this step lies in the fact that it allows us to work with the linear form

$$
\Lambda_{1}=\log \omega^{(1)}=b_{2} \log \eta_{2}^{(1)}-b_{1} \log \eta_{1}^{(1)}
$$

in two logarithms of real algebraic numbers, instead of with a linear form in three logarithms of complex algebraic numbers, the third being a logarithm $\pi i$ of -1 , as would have been the case if we had chosen an $\omega$ close to 1 at the complex place. For linear forms in two logarithms like $\Lambda_{1}$, the lower bounds offered by transcendence theory are considerably sharper than for three or more logarithms. As we shall see, moving $\omega$ into the vicinity of 1 at the first real place is sufficient to keep it away from 1 at the other two places. Note that this does not generalize to fields of larger unit rank, but it is always possible, using $\mathcal{H}$, to move an exceptional unit away from 1 (more precisely, to move it out of the subset $|z-1|<1, \Re z>1 / 2$ of $\mathbb{C}$ ) at two prescribed places.

Note for future reference the a priori bounds

$$
0<\Lambda_{1}<\log 2 .
$$

Lemma 1. There are no nontrivial solutions with $b_{1}=0$.

Proof. We need rough estimates for each of the embeddings of $\eta_{2}$; they give a taste of what will follow in the next section.

When $a=1$, we find $1.269<\eta_{2}^{(1)}<1.270$ and $\eta_{2}^{(2)}>2.665$, whereas $\left|\eta_{2}^{(3)}\right|<0.55$. Thus $\left(\eta_{2}^{b_{2}}-1\right)^{(1)}>0.610$ for $b_{2} \geq 2$, as well as $\left|\left(\eta_{2}^{b_{2}}-1\right)^{(3)}\right|>0.6975$ and so $N_{K / \mathbb{Q}}\left(\eta_{2}^{b_{2}}-1\right)>1.81$ if $b_{2} \geq 2$. Hence the lemma follows in the case $a=1$.

Now suppose $a>1$. The minimal polynomial of $\eta_{2}$ is

$$
g_{a}(x)=x^{4}-\left(a^{2}+2\right) x^{3}+\left(a^{2}-1\right) x^{2}+2 x+1 .
$$


Its value at $x=a^{2}+1$ is

$$
-2 a^{4}-2 a^{2}+1
$$

which is negative for all $a \geq 1$, hence $\eta_{2}^{(2)}>a^{2}+1$. Furthermore, $g_{a}\left(1+a^{-2} / 2\right)$ is positive for $a \geq 2$, showing that $\eta_{2}^{(1)}-1>1 / 2 a^{2}$. Since $\eta_{2}$ is a unit,

$$
\left|\eta_{2}^{(3)}\right|^{2}=\left(\eta_{2}^{(1)} \eta_{2}^{(2)}\right)^{-1}<1 / a^{2} \leq 1 / 4
$$

when $a \geq 2$, and we conclude in this case that for all integers $b_{2} \geq 2$,

$$
\begin{aligned}
N_{K / \mathbb{Q}}\left(\eta_{2}^{b_{2}}-1\right) & =\left(\eta_{2}^{b_{2}}-1\right)^{(1)} \cdot\left(\eta_{2}^{b_{2}}-1\right)^{(2)} \cdot\left|\left(\eta_{2}^{b_{2}}-1\right)^{(3)}\right|^{2} \\
& >\left(\left(1 / a^{2}+1\right)^{b_{2}}-1\right) \cdot\left(\left(a^{2}+1\right)^{b_{2}}-1\right) \cdot(3 / 4)^{2} \\
& \geq(9 / 16) a^{2 b_{2}-2}>1 .
\end{aligned}
$$

Hence $\eta_{2}^{b_{2}}-1$ does not have norm one and so $\eta_{2}^{b_{2}}$ can never be an exceptional unit.

\section{BRACKETS FOR THE LOGARITHMS OF THE GENERATOR UNITS}

Using a formal Newton-Raphson iteration, it is easy to obtain series expansions in powers of $a^{-1}$ for the real roots of $f_{a}$ and of $g_{a}$, and then, by splitting them into a dominant factor and a series starting with 1 , to deduce series expansions of their logarithms. The following lemma shows that initial pieces of the series belonging to the first real place are well suited for bounding the corresponding logarithms from both sides.

Lemma 2. Consider the two series expansions

$$
\begin{aligned}
& S_{1}=2 a^{-2}-7 a^{-4}+\frac{113}{3} a^{-6}-\frac{485}{2} a^{-8}+\frac{8612}{5} a^{-10}-\frac{78095}{6} a^{-12}+\frac{718577}{7} a^{-14} \\
& S_{2}=a^{-2}-\frac{9}{2} a^{-4}+\frac{79}{3} a^{-6}-\frac{705}{4} a^{-8}+\frac{6396}{5} a^{-10}-\frac{19597}{2} a^{-12}+\frac{545917}{7} a^{-14} .
\end{aligned}
$$

For $0 \leq k \leq 7$ and $j \in\{1,2\}$, let $S_{j}^{k}$ denote the result of truncating $S_{j}$ after the term proportional to $a^{-2 k}$; in particular, $S_{j}^{0}=0$. Then, for all $a \geq 3$,

$$
\operatorname{sgn}\left(\log \eta_{1}^{(1)}-\left(\log a+S_{1}^{k}\right)\right)=\operatorname{sgn}\left(\log \eta_{2}^{(1)}-S_{2}^{k}\right)=(-1)^{k}
$$

Proof. It is easily checked that $0 \leq S_{j}^{k}<1$ for all $a, j, k$ under consideration. Therefore the value of $\exp \left(-S_{j}^{k}\right)$ is sandwiched between every pair of successive partial sums $E_{j}^{k, n}=\sum_{\ell=0}^{n}\left(-S_{j}^{k}\right)^{\ell} / \ell$ ! of the exponential series. To prove the lemma, compute $E_{j}^{k, k+1}$ as a Laurent polynomial (rational linear combination of finitely many powers of $a$ ), substitute $\left(a E_{1}^{k, k+1}\right)^{-1}$ into $f_{a}$ and $E_{2}^{k, k+1}$ into $g_{a}$, and examine the signs of the resulting expressions, which turn out to be the right ones for all $a \geq 3$.

(A computer algebra package is indispensable here, and instead of substituting rational functions of $a$ into the polynomials, one should substitute their numerators and denominators into the associated homogeneous binary forms - this can speed up computation times from many hours to a few seconds. Even so, this step probably requires more machine time than all the diophantine computations of section 6 .) 
Similar but simpler reasoning, still assuming $a \geq 3$, establishes the brackets

$$
\begin{aligned}
-\log a-a^{-4} & <\log \left|\eta_{1}^{(2)}\right|<-\log a, \\
2 \log a & <\log \eta_{2}^{(2)}<2 \log a+2 a^{-2} .
\end{aligned}
$$

Combining this with $S_{1}^{2}$ and $S_{1}^{1}$ on the one hand, and with $S_{2}^{0}$ and $S_{2}^{1}$ on the other, we deduce from $\left|N_{K / \mathbb{Q}}\left(\eta_{j}\right)\right|=1$ that

$$
\begin{aligned}
-2 a^{-2} & <\log \left|\eta_{1}^{(3)}\right|^{2}<-2 a^{-2}+8 a^{-4}, \\
-2 \log a-3 a^{-2} & <\log \left|\eta_{2}^{(3)}\right|^{2}<-2 \log a .
\end{aligned}
$$

We supplement the preceding results with numerical intervals enclosing the six logarithms for small values of $a$. Although $3 \leq a \leq 5$ are handled by the above, it will be profitable to have sharper estimates for this range. The shorthand notation for intervals should be self-explaining. For later use we also record brackets for $\beta=\log \eta_{1}^{(1)} / \log \eta_{2}^{(1)}$.

\begin{tabular}{|c|l|l|l|l|l|}
\hline$a$ & 1 & \multicolumn{1}{|c|}{2} & \multicolumn{1}{c|}{3} & \multicolumn{1}{c|}{4} & \multicolumn{1}{c|}{5} \\
\hline $\log \eta_{1}^{(1)}$ & $+0.65625_{59}^{60}$ & $+0.992064_{8}^{9}$ & $+1.265138_{7}^{8}$ & $+1.490567_{3}^{4}$ & $+1.680163_{5}^{6}$ \\
$\log \eta_{2}^{(1)}$ & $+0.23834_{19}^{20}$ & $+0.128833_{0}^{1}$ & $+0.076624_{79}^{80}$ & $+0.0494901_{4}^{5}$ & $+0.0341346_{2}^{3}$ \\
$\beta$ & $+2.75342_{11}^{28}$ & $+7.7003_{87}^{95}$ & $+16.5108_{2}^{3}$ & $+30.1184_{6}^{8}$ & $+49.2216_{7}^{9}$ \\
\hline $\log \left|\eta_{1}^{(2)}\right|$ & $-0.255145_{1}^{0}$ & $-0.736790_{6}^{5}$ & $-1.109336_{8}^{7}$ & $-1.38992_{60}^{59}$ & $-1.610968_{4}^{3}$ \\
$\log \eta_{2}^{(2)}$ & $+0.980494_{5}^{6}$ & $+1.679866_{1}^{2}$ & $+2.321909_{7}^{8}$ & $+2.840050_{7}^{8}$ & $+3.261039_{8}^{9}$ \\
\hline $\log \left|\eta_{1}^{(3)}\right|$ & $-0.200555_{5}^{4}$ & $-0.127637_{2}^{1}$ & $-0.07790_{100}^{099}$ & $-0.0503207_{1}^{0}$ & $-0.0345976_{1}^{0}$ \\
$\log \left|\eta_{2}^{(3)}\right|$ & $-0.609418_{3}^{2}$ & $-0.904349_{7}^{6}$ & $-1.199267_{3}^{2}$ & $-1.444770_{5}^{4}$ & $-1.647587_{3}^{2}$ \\
\hline
\end{tabular}

Lemma 3. The units $\eta_{1}$ and $\eta_{2}$ are multiplicatively independent.

Proof. The unit subgroup regulator of $G$ can be expressed in several ways as the absolute value of a determinant of four logarithms from among $\log \left|\eta_{j}^{(1,2)}\right|$ and $\log \left|\eta_{j}^{(3)}\right|^{2}$. It suffices to show that any one of these determinants is nonzero, which is easy using (3)-(6) for $a \geq 3$ and the entries of the preceding table for $a \in\{1,2\}$.

In what follows, notations of the form $c_{i}(a)$ with $i=1,2, \ldots$ will always denote positive real-valued functions of $a$.

Recall that the absolute logarithmic height $h(\alpha)$ of a nonzero algebraic integer $\alpha$ is the sum over $\max \left\{0, \log \left|\alpha^{(k)}\right|\right\}$ divided by the degree $d$ of $\mathbb{Q}(\alpha)$ over $\mathbb{Q}$, where $\alpha^{(k)}$ ranges over all $d$ embeddings of $\alpha$ into $\mathbb{C}$. Evaluating this for $\alpha=\eta_{1}$ and $\alpha=\eta_{2}$ in turn gives, using (3)-(6) and again $\log a+S_{1}^{1}$ and $S_{2}^{1}$ as upper bounds for the $\log \eta_{j}^{(1)}$,

Lemma 4. For $a \geq 6$, let $c_{1}(a)=\log a+2 a^{-2}$. The absolute logarithmic heights of $\eta_{1}$ and $\eta_{2}$ satisfy

$$
\begin{aligned}
& 4 h\left(\eta_{1}\right)<c_{1}(a), \\
& 4 h\left(\eta_{2}\right)<2 c_{1}(a) .
\end{aligned}
$$


The second bound has been chosen slightly weaker than optimal in order to simplify our formulas in section 5. This makes little difference for $a \geq 6$. For smaller $a$, we compute better values from $(7)$ :

\begin{tabular}{|c|c|c|c|c|c|}
\hline$a$ & 1 & 2 & 3 & 4 & 5 \\
\hline $4 h\left(\eta_{1}\right)<$ & 0.6563 & 0.9921 & 1.2652 & 1.4906 & 1.6802 \\
\hline $4 h\left(\eta_{2}\right)<$ & 1.2189 & 1.8087 & 2.3986 & 2.8896 & 3.2952 \\
\hline
\end{tabular}

\section{The gap and diophantine approximation inequalities}

The fact that $\eta_{2}$ is an exceptional unit of fairly large height, and thus fairly close to 1 at the first real place, whereas $-\eta_{1}^{-2}$ is the complementary unit $1-\eta_{2}$ and therefore fairly close to zero at that place, is responsible for the very disparate sizes of the logarithms of $\eta_{1}^{(1)}$ and $\eta_{2}^{(1)}$. These in turn force the exponents involved in any nontrivial solutions to be rather large. More precisely, the trivial bounds on $\Lambda_{1}$ from (2) suffice to show that the rational number $b_{2} / b_{1}$, which "tries to" approximate $\beta=\log \eta_{1}^{(1)} / \log \eta_{2}^{(1)}$, must also be very large, indeed of size exponential in the height of the trivial solutions. Since $b_{1}$ is a nonzero integer by Lemma $1,\left|b_{2}\right|$ must be very large indeed. We call this a "gap inequality", alluding to far more general "gap principles" of which this is a special and explicit instance.

Lemma 5 (Gap Inequality, preliminary form). If $b_{1}, b_{2}$ come neither from a trivial solution nor from the known nontrivial ones with $a=1$,

$$
\left|b_{2}\right| \geq \frac{b_{2}}{b_{1}}>c_{2}(a)
$$

where for $a \geq 6$,

$$
c_{2}(a)=a^{2} \log (a / 2)>39.550
$$

and for smaller a we use the values

\begin{tabular}{|c|c|c|c|c|c|}
\hline$a$ & 1 & 2 & 3 & 4 & 5 \\
\hline$c_{2}(a)$ & 1.2993 & 2.3201 & 7.4648 & 16.112 & 28.915 \\
\hline
\end{tabular}

derived from (7). Furthermore, $\left|b_{2}\right| \geq 3$ even for $a=1$ apart from the known nontrivial solution.

Proof. First notice that if $a=1$ and $\left|b_{1}\right| \leq 1$, then from (2) we have $\left|b_{2}\right| \leq 5$, and if $\left|b_{2}\right| \leq 2$, then again from (2) we have $\left|b_{1}\right| \leq 1$. It is easy to determine that the only exceptional units satisfying these inequalities are the trivial and the known non-trivial ones. Hence we shall now assume that either $a \geq 2$ or $a=1$ and $\left|b_{1}\right|>1$ and $\left|b_{2}\right|>2$.

Supposing first that $b_{1}>0$, we have

$$
\frac{b_{2}}{b_{1}}=\frac{\Lambda_{1}}{b_{1} \log \eta_{2}^{(1)}}+\beta>\beta
$$

since $\Lambda_{1}>0$ and $\log \eta_{2}^{(1)}>0$. For $a<6$ we notice that $\beta$ is larger than the values in the table above. For $a \geq 6$ we use Lemma 2 with $S_{1}^{0}$ and $S_{2}^{1}$ to deduce

$$
\beta=\frac{\log \eta_{1}^{(1)}}{\log \eta_{2}^{(1)}}>\frac{\log a}{a^{-2}}>c_{2}(a)
$$

as required. 
Now suppose that $b_{1}<0$. Then

$$
-\frac{b_{2}}{b_{1}}=\frac{\Lambda_{1}}{\left|b_{1}\right| \log \eta_{2}^{(1)}}-\beta<\frac{\log 2}{\left|b_{1}\right| \log \eta_{2}^{(1)}}-\beta \leq \frac{\log 2}{\log \eta_{2}^{(1)}}-\beta
$$

since $\Lambda_{1} \leq \log 2$. Hence

$$
\frac{b_{2}}{b_{1}}>\beta-\frac{\log 2}{\log \eta_{2}^{(1)}} .
$$

For all $a \geq 6$ we then find, again using Lemma 2, that

$$
\frac{b_{2}}{b_{1}}>\left(\log \eta_{1}^{(1)}-\log 2\right) / \log \eta_{2}^{(1)}>\frac{\log a-\log 2}{a^{-2}}=c_{2}(a) .
$$

When $2 \leq a \leq 5$ we find that $\left(\log \eta_{1}^{(1)}-\log 2\right) / \log \eta_{2}^{(1)}$ is always greater than or equal to the value in the table above. Hence we are only left with the case $a=1$, but our earlier assumptions $\left|b_{1}\right|>1$ and $\left|b_{2}\right|>2$ for this case give $\frac{b_{2}}{b_{1}}>$ $\beta-\log 2 /\left(2 \log \eta_{2}^{(1)}\right)>1.2993$.

One consequence of this inequality is that $b_{1}$ and $b_{2}$ must have the same sign. This gives us sufficient control at the other two archimedean places to show that one of them will contribute a very large factor to the absolute norm of $\omega-1$ and the other will contribute a factor not much less than unity.

Lemma 6 (Diophantine Approximation Inequality). Any further exceptional unit would have to satisfy

$$
\log \Lambda_{1}<-c_{3}(a)\left|b_{2}\right|
$$

where $c_{3}(a)=1.9992 \log a$ for $a \geq 6$, implying $\log \Lambda_{1}<-143$, and the values for small a are found in the following table:

\begin{tabular}{|c|c|c|c|c|c|}
\hline$a$ & 1 & 2 & 3 & 4 & 5 \\
\hline$c_{3}(a)$ & 0.6954 & 1.6232 & 2.3218 & 2.8399 & 3.2609 \\
\hline$\Lambda_{1}<$ & 0.1242 & 0.0077 & $10^{-8}$ & $10^{-20}$ & $10^{-41}$ \\
\hline
\end{tabular}

Proof. In the following estimates we will use the elementary facts that for fixed positive $c$ and positive $x$, the expression $x^{-1} \log (1-\exp (-c x))$ is a monotonically increasing function of $x$ and satisfies

$$
0>\frac{\log (1-\exp (-c x))}{x}>\frac{-1}{x(\exp (c x)-1)} .
$$

The proof is split into two cases depending on whether $b_{1}, b_{2}>0$ or $b_{1}, b_{2}<0$.

When $b_{1}, b_{2}>0$, we have

$$
\Lambda_{2}=\log \left|\omega^{(2)}\right|=b_{2} \log \eta_{2}^{(2)}-b_{1} \log \left|\eta_{1}^{(2)}\right|>b_{2} \log \eta_{2}^{(2)}
$$

and

$$
\begin{aligned}
\log \left|\omega^{(2)}-1\right| & \geq \log \left(\exp \Lambda_{2}-1\right)=\Lambda_{2}+\log \left(1-\exp \left(-\Lambda_{2}\right)\right) \\
& >b_{2} \log \eta_{2}^{(2)}-\frac{1}{\exp \Lambda_{2}-1} \\
& \geq b_{2}\left(\log \eta_{2}^{(2)}-\frac{1}{b_{2}\left(\left(\eta_{2}^{(2)}\right)^{b_{2}}-1\right)}\right) .
\end{aligned}
$$


For the third conjugate we find, using Lemma 5 to bound $b_{1} / b_{2}$,

$$
\begin{aligned}
\Lambda_{3}=\log \left|\omega^{(3)}\right| & =b_{2} \log \left|\eta_{2}^{(3)}\right|-b_{1} \log \left|\eta_{1}^{(3)}\right| \\
& =-b_{2}\left(|\log | \eta_{2}^{(3)}||-\frac{b_{1}}{b_{2}}|\log | \eta_{1}^{(3)}||\right) \\
& \leq-b_{2}\left(|\log | \eta_{2}^{(3)}||-\frac{|\log | \eta_{1}^{(3)}||}{c_{2}(a)}\right)=-b_{2} F(a),
\end{aligned}
$$

say, and so

$$
\begin{aligned}
\log \left|\omega^{(3)}-1\right|^{2} & \geq 2 \log \left(1-\exp \Lambda_{3}\right)>b_{2} \frac{-2}{b_{2}\left(\exp \left(-\Lambda_{3}\right)-1\right)} \\
& \geq b_{2} \frac{-2}{b_{2}\left(\exp \left(b_{2} F(a)\right)-1\right)} .
\end{aligned}
$$

Hence

$$
\begin{aligned}
\log \Lambda_{1} & <\log \left(\exp \Lambda_{1}-1\right)=\log \left(\omega^{(1)}-1\right)=-\log \left|\omega^{(2)}-1\right|-\log \left|\omega^{(3)}-1\right|^{2} \\
& \leq-b_{2}\left(\log \eta_{2}^{(2)}-\frac{1}{b_{2}\left(\left(\eta_{2}^{(2)}\right)^{b_{2}}-1\right)}-\frac{2}{b_{2}\left(\exp \left(b_{2} F(a)\right)-1\right)}\right) .
\end{aligned}
$$

The proof for this case is concluded by using the lower bound $b_{2} \geq c_{2}(a)$ from Lemma 5 and using (3)-(6) to control the $\log \left|\eta_{j}^{(k)}\right|$ 's and thus $F(a)$.

When $b_{1}, b_{2}<0$, we have

$$
\Lambda_{2}=\log \left|\omega^{(2)}\right| \leq-\left|b_{2}\right| \log \eta_{2}^{(2)}
$$

and

$$
\begin{aligned}
\log \left|\omega^{(2)}-1\right| & =\log \left(1-\exp \left(\Lambda_{2}\right)\right)>\frac{-1}{\exp \left(-\Lambda_{2}\right)-1} \\
& >\frac{-1}{\eta_{2}^{(2)}\left|b_{2}\right|-1} .
\end{aligned}
$$

The lower bound on $\log \left|\omega^{(3)}-1\right|$ again involves the expression $F(a)$. We have

$$
\Lambda_{3} \geq\left|b_{2}\right|\left(|\log | \eta_{2}^{(3)}||-\frac{|\log | \eta_{1}^{(3)}||}{c_{2}(a)}\right)
$$

and therefore

$$
\begin{aligned}
\log \left|\omega^{(3)}-1\right|^{2} & \geq 2 \log \left(\exp \Lambda_{3}-1\right)=2 \Lambda_{3}+2 \log \left(1-\exp \left(-\Lambda_{3}\right)\right) \\
& >2\left(\Lambda_{3}-\frac{1}{\exp \Lambda_{3}-1}\right) .
\end{aligned}
$$

The second expression in the parentheses is nothing but $\left(\exp \left(-\Lambda_{3}^{\prime}\right)-1\right)^{-1}$ where $\Lambda_{3}^{\prime}$ arises from $\Lambda_{3}$ by changing the signs of $b_{1}$ and of $b_{2}$, and we have already obtained a lower bound for the denominator while considering the previous case. Thus

$$
\log \left|\omega^{(3)}-1\right|^{2} \geq 2\left|b_{2}\right|\left(|\log | \eta_{2}^{(3)}||-\frac{|\log | \eta_{1}^{(3)}||}{c_{2}(a)}-\frac{1}{\left|b_{2}\right|\left(\exp \left(\left|b_{2}\right| F(a)\right)-1\right)}\right) .
$$

The assertion now follows from $\log \Lambda_{1}<-\log \left|\omega^{(2)}-1\right|-\log \left|\omega^{(3)}-1\right|^{2}$ as before. 
When we proved the first version of the gap inequality, we had used $\Lambda_{1}<\log 2$ to bound $\left|b_{2} / b_{1}-\beta\right|$, obtaining essentially $(\log 2) / a^{2}$. We can now replace $\log 2$ with the upper bound on $\Lambda_{1}$ which results from substituting the old lower bounds for $\left|b_{2}\right|$ into the approximation inequality.

Lemma 7 (Gap Inequality, sharp form). If $b_{1}, b_{2}$ come neither from a trivial solution nor from the obvious nontrivial ones for $a=1$,

$$
\left|b_{2}\right| \geq \frac{b_{2}}{b_{1}}>c_{4}(a)
$$

where for $a \geq 6$,

$$
c_{4}(a)=\left(a^{2}+4\right) \log a+2>73.67
$$

and for smaller a we use the values

\begin{tabular}{|c|c|c|c|c|c|}
\hline$a$ & 1 & 2 & 3 & 4 & 5 \\
\hline$c_{4}(a)$ & 2.5797 & 7.6803 & 16.510 & 30.118 & 49.221 \\
\hline
\end{tabular}

Proof. The generic case $(a \geq 6)$ is, using $S_{1}^{2}$ and $S_{2}^{3}$ from Lemma 2,

$$
\begin{aligned}
\frac{b_{2}}{b_{1}} & >\frac{\log \eta_{1}^{(1)}-\Lambda_{1} /\left|b_{2}\right|}{\log \eta_{2}^{(1)}} \\
& >a^{2} \frac{\log a+2 a^{-2}-7 a^{-4}-10^{-60}}{1-(9 / 2) a^{-2}+(79 / 3) a^{-4}} \\
& >\left(a^{2}+4\right) \log a+2,
\end{aligned}
$$

and we leave the calculations for the small parameter values to the reader.

This procedure of using the bounds on $\Lambda_{1}$ and the gap inequality to sharpen each other could be iterated yet again, but the profit would be marginal.

\section{BOUNDING A LINEAR FORM IN TWO LOGARITHMS}

We shall now apply a lower bound tailored to linear forms in the logarithms of two multiplicatively independent, positive real algebraic numbers $\alpha_{1}$ and $\alpha_{2}$, due to Laurent, Mignotte and Nesterenko [4], where one can also find much more general results. We paraphrase their Corollaire 2 almost verbatim, except for the following three modifications: The numerical coefficients have been replaced with those given in the last entry of Tableau 2 of [4, section 8], the equality relating the quantity $b^{\prime}$ to $b_{1}$ and $b_{2}$ has been weakened to an inequality for ease of use, and we write $\left|b_{j}\right|$ instead of assuming that the $b_{j}$ are positive. (They are still supposed to be nonzero.) The form $\Lambda_{1}$ is as we had defined it in (1) above, and $h\left(\eta_{j}\right)$ denotes the absolute logarithmic height as in our Lemma 4. Let

$$
D=\left[\mathbb{Q}\left(\alpha_{1}, \alpha_{2}\right): \mathbb{Q}\right],
$$

and let $A_{1}, A_{2} \in \mathbb{R}$ denote two positive real numbers such that

$$
D \log A_{j} \geq \max \left\{D h\left(\alpha_{j}\right),\left|\log \alpha_{j}\right|, 1\right\}
$$

and $b^{\prime}$ a positive number satisfying

$$
b^{\prime} \geq \frac{\left|b_{1}\right|}{D \log A_{2}}+\frac{\left|b_{2}\right|}{D \log A_{1}} .
$$


Theorem (Laurent, Mignotte and Nesterenko). Under the above conditions, we have

$$
\log \left|\Lambda_{1}\right| \geq-22.13 D^{4}\left(\max \left\{\log b^{\prime}+0.71, \frac{30}{D}, \frac{1}{2}\right\}\right)^{2} \log A_{1} \log A_{2} .
$$

We will take $\alpha_{j}=\eta_{j}$, thus $D=4$, and the $\frac{1}{2}$ inside the maximum is of no significance. The middle term of the maximum is dominated by the first when $\log b^{\prime} \geq 6.79$; the upper bound on $\log b^{\prime}$ which we shall derive using the first term will be larger than that, so we may also drop the middle term.

Using Lemma 4 , we see that $D \log A_{1}$ can be taken to be $\log a+2 a^{-2}$ for $a \geq 6$ (all of the height comes from the logarithm under consideration), and for $D \log A_{2}$ we may take twice this amount. Then we set

$$
b^{\prime}=c_{5}(a)\left|b_{2}\right|
$$

where $c_{5}(a)=1.0068 / \log a$; that this satisfies the required inequality follows at once from Lemma 7. For $1 \leq a \leq 5$, we use the tables after Lemma 4 and in Lemma 7 to justify the following choices for $c_{5}(a)$, taking the opportunity to list also the numerical coefficients $c_{6}(a)=22.13 D^{2} \cdot D \log A_{1} \cdot D \log A_{2}$ :

\begin{tabular}{|r|c|c|c|c|c|}
\hline$a$ & 1 & 2 & 3 & 4 & 5 \\
\hline$D \log A_{1}$ & 1 & 1 & 1.2652 & 1.4906 & 1.6802 \\
\hline$D \log A_{2}$ & 1.2189 & 1.8087 & 2.3986 & 2.8896 & 3.2952 \\
\hline$c_{5}(a)$ & 1.3181 & 1.0720 & 0.8157 & 0.6824 & 0.6014 \\
\hline$c_{6}(a)$ & 431.59 & 640.43 & 1074.6 & 1525.2 & 1960.4 \\
\hline
\end{tabular}

The estimate now reads

$$
\log \Lambda_{1} \geq \begin{cases}-708.16\left(\log a+2 a^{-2}\right)^{2}\left(\log b^{\prime}+0.71\right)^{2}, & a \geq 6, \\ -c_{6}(a)\left(\log b^{\prime}+0.71\right)^{2}, & \text { always. }\end{cases}
$$

We shall apply this twice, first to obtain an upper bound on $a$ and then to bound $\left|b_{2}\right|$ for any fixed value of $a$.

Proposition 1. Further solutions can only exist when $a \leq 215$.

Proof. Assuming $a \geq 6$, then combining (9) with the approximation inequality from Lemma 6 , we obtain

$$
-c_{6}(a)\left(\log b^{\prime}+0.71\right)^{2} \leq \log \Lambda_{1}<-c_{3}(a)\left|b_{2}\right| .
$$

We now substitute $b^{\prime}=c_{5}(a)\left|b_{2}\right|$ into this last equation, obtaining

$$
c_{6}(a)\left(\log \left(c_{5}(a)\left|b_{2}\right|\right)+0.71\right)^{2}-c_{3}(a)\left|b_{2}\right|>0 .
$$

For fixed $a$, the left-hand side is a monotonically decreasing function of $\left|b_{2}\right|$ provided that $a \geq 83$ and that $\left|b_{2}\right|$ satisfies the gap inequality $\left|b_{2}\right|>c_{4}(a)\left|b_{1}\right| \geq c_{4}(a)$ or its weaker consequence $\left|b_{2}\right|>a^{2} \log a$. Note that these conditions ensure that $b^{\prime}>6.79$ by a wide margin. It is therefore sufficient to show that the left-hand side with $\left|b_{2}\right|$ replaced by $a^{2} \log a$ becomes negative when $a$ is large enough. The expression in question, with $\log 1.0068+0.71$ replaced by the larger quantity 0.72 , and then divided by $(\log a)^{4}$, reads

$$
708.16\left(1+\frac{2}{a^{2} \log a}\right)^{2}\left(2+\frac{0.72}{\log a}\right)^{2}-1.9992 \frac{a^{2}}{(\log a)^{2}},
$$


which is a monotonically decreasing function of $a$ for $a \geq 3$, and becomes negative at $a=216$. (Even the full strength of the gap inequality does not yield a smaller result here, but it will help in the next section.)

The second application is even simpler.

Proposition 2. Nontrivial solutions, other than the known ones with $a=1$, can occur only when $\left|b_{2}\right| \leq c_{7}(a)$, where $c_{7}(a)=47348.1 \log a$ for $a \geq 11$ and its values for smaller a are as follows:

\begin{tabular}{|c|c|c|c|c|c|}
\hline$a$ & 1 & 2 & 3 & 4 & 5 \\
\hline$c_{7}(a)$ & 96396 & 53750 & 61579 & 71014 & 79265 \\
\hline \hline$a$ & 6 & 7 & 8 & 9 & 10 \\
\hline$c_{7}(a)$ & 89837 & 95285 & 100398 & 105125 & 109503 \\
\hline
\end{tabular}

Proof. Recall that we need to assume $b^{\prime}>\exp 6.79>888.9$ before we can apply (9). Eliminate $b_{2}$ in favour of $b^{\prime}$ from the combination of (9) with the approximation inequality and divide by $(\log a)^{2}$ to obtain

$$
708.16\left(1+\frac{2}{a^{2} \log a}\right)^{2}\left(\log b^{\prime}+0.71\right)^{2}-\frac{1.9992}{1.0068} b^{\prime}>0 .
$$

The product of the first two factors on the left-hand side is less than

\begin{tabular}{|c|c|c|c|c|c|c|}
\hline$a$ & 6 & 7 & 8 & 9 & 10 & $\geq 11$ \\
\hline & 752.76 & 738.18 & 729.61 & 724.17 & 720.52 & 717.96 \\
\hline
\end{tabular}

The left-hand side is a monotonically decreasing function of $b^{\prime}$ when $a$ is fixed and $\log b^{\prime}>6.79$ (in fact already for rather smaller $b^{\prime}$ ), and it becomes negative when $b^{\prime}$ exceeds

\begin{tabular}{|c|c|c|c|c|c|c|}
\hline$a$ & 6 & 7 & 8 & 9 & 10 & $\geq 11$ \\
\hline & 50480 & 49300 & 48610 & 48170 & 47880 & 47670 \\
\hline
\end{tabular}

Hence (for instance) if $a \geq 11$ then $\left|b_{2}\right| \leq 47670 / c_{5}(a) \leq 47348.1 \log a$.

This leaves us with the small values of $a$, for which the necessary condition reads: Either $b^{\prime}<889$ or

$$
c_{6}(a)\left(\log b^{\prime}+0.71\right)^{2}-\frac{c_{3}(a)}{c_{5}(a)} b^{\prime}>0 .
$$

Checking monotonicity of the left-hand side each time (valid for $b^{\prime} \geq 17200$ in the worst case $a=1$, earlier for $2 \leq a \leq 5$ ) and looking for the change of sign, we find the following upper limits on $b^{\prime}$ :

\begin{tabular}{|c|c|c|c|c|c|}
\hline$a$ & 1 & 2 & 3 & 4 & 5 \\
\hline & 127060 & 57620 & 50230 & 48460 & 47670 \\
\hline
\end{tabular}

(The apparent non-monotonicity of the results between $a=5$ and $a=6$ is spurious; it is mainly caused by the fact that our generic $D \log A_{2}$ is not very sharp for $a=6$.) Rewriting (11) and (10) in terms of $\left|b_{2}\right|$, the result follows. 


\section{Closing the gaP}

Even more interesting than the upper bounds on $\left|b_{2}\right|$ are the resulting upper bounds on $\left|b_{1}\right|$, obtained by combining Proposition 2 with the gap inequality of Lemma 7.

$$
\left|b_{1}\right|<\frac{\left|b_{2}\right|}{c_{4}(a)}<\frac{47348.1 \log a}{c_{4}(a)} \text { if } a \geq 11
$$

Two immediate consequences are that for $154 \leq a \leq 215$, only $\left|b_{1}\right|=1$ is possible, and for $a \geq 66$ the exponent $b_{1}$ is restricted to $\left|b_{1}\right| \leq 10$.

Since Lemma 6 imposes $\Lambda_{1}<\exp (-143)$ on any nontrivial solutions with $a \geq 6$, which implies $\left|\beta-b_{2} / b_{1}\right|<\exp (-132)<10^{-57}$ for $6 \leq a \leq 215$, we only need to check that $\beta$ is not extremely close to a rational number of denominator not exceeding 10 for $66 \leq a \leq 125$, to half an integer for $126 \leq a \leq 153$, and to an integer for $154 \leq a \leq 215$ in order to exclude nontrivial solutions in this entire range. Instead of inspecting an approximate value of $\beta$, one should of course work with upper and lower bounds. Such bounds are readily obtained from Lemma 2 using $S_{1}^{3}, S_{1}^{4}, S_{2}^{3}$ and $S_{2}^{4}$ in appropriate combinations. Ideally these bounds should be computed with directed rounding in every arithmetic operation, but if the individual results are just rounded correctly to the nearest representable machine number, one can estimate the maximal accumulated error and then add a suitable quantity to the upper bound and subtract it from the lower bound. No candidates for solutions are found here.

(Ten-digit pocket-calculator accuracy is almost sufficient to cover this range; with more significant digits available, like the default PARI/GP precision of about 28 decimal places, one can exploit the shortcut of testing whether $2520 \beta$ is close to an integer for $66 \leq a \leq 125$.)

For $a \leq 65$, testing individual denominator candidates becomes unattractive. Instead, we invoke the ordinary continued fraction expansion of $\beta$. Note first that Lemmas 2, 6 and 7 together imply

$$
\left|\beta-b_{2} / b_{1}\right|<\frac{1}{2\left|b_{1}\right|^{2}} \cdot \frac{2 a^{2}\left|b_{1}\right|}{\left(1-\frac{9}{2} a^{-2}\right) \exp \left(c_{3}(a) a^{2} \log a\left|b_{1}\right|\right)}
$$

when $a \geq 3$; the second factor is less than $4\left|b_{1}\right| / 10^{9\left|b_{1}\right|}$ and thus far smaller than 1 . Therefore $b_{2} / b_{1}$ is a convergent of the continued fraction expansion of $\beta$. If $b_{1}$ and $b_{2}$ had a common factor $b_{0}>1$, then our putative nontrivial exceptional unit, $\omega$, would be of the form $\omega_{0}^{b_{0}}$ with a unit $\omega_{0} \in G$, and $\omega_{0}-1$, which divides $\omega-1$, would also be a unit - in other words, $\omega_{0}$ itself would have to be a nontrivial solution. Therefore it is no loss of generality to assume that $b_{1}$ and $b_{2}$ are coprime. But then $\left|b_{2}\right|$ and $\left|b_{1}\right|$ itself are the numerator $p_{n}$ and the denominator $q_{n}$ of a continued fraction convergent of $\beta$.

This argument remains valid for $a=2$ and also for $a=1$ when $\left|b_{1}\right|>1$, except for the elementary verification that proper powers of the known nontrivial solutions for $a=1$ do not produce any further solutions, when the factor $S_{2}^{2}$ coming from the $\log \eta_{2}^{(1)}$ in the denominator is replaced with a tight numerical estimate for that logarithm, and $a^{2} \log a$ under the exponential with the explicit values $c_{4}(1)$ and $c_{4}(2)$. 
Moreover, when $\left|\beta-b_{2} / b_{1}\right|=\left|\beta-p_{n} / q_{n}\right|$ is smaller than $1 /\left(2 q_{n}^{2}\right)$ we have an explicit lower bound for the next partial denominator $a_{n+1}$ :

$$
a_{n+1}+2>\left(q_{n}^{2}\left|\beta-\frac{p_{n}}{q_{n}}\right|\right)^{-1}
$$

(This is an easy consequence of Theorems 163 and 171 of [3].) It implies $a_{n+1}>10^{8}$ for any nontrivial solution with $a \geq 3$, and $a_{n+1}>10^{4}$ for any with $a=2$. For $a=1$, it implies $a_{n+1} \geq 37$ when $\left|b_{1}\right| \geq 4$ and $a_{n+1}>180$ for $\left|b_{1}\right|>4$; we won't need to consider $\left|b_{1}\right| \in\{2,3\}$ since the sequence of denominators of convergents begins $1,1,4,18, \ldots$ in this case.

It remains to verify for each $1 \leq a \leq 65$ that no partial denominators of the indicated size occur in the expansion of $\beta$ before the denominators of the convergents exceed the upper bound on $\left|b_{1}\right|$. The expansions should be computed using Lehmer's technique [1, Algorithm 1.3.13] of simultaneously expanding an upper and a lower bound for the true value of $\beta$. The partial denominators in common to both expansions are then known to be correct, and the first discordant pair of partial denominators still yields rigorous bounds for the correct value. As before, suitable bounds for $\beta$ can be obtained from Lemma 2 when $a$ is not too small. The same truncation orders $a^{-6}$ and $a^{-8}$ as used earlier are sufficient for $a \geq 50$. For $a \leq 49$, the truncated series $S_{1}^{3}$ and $S_{2}^{3}$ should be replaced with $S_{1}^{5}$ and $S_{2}^{5}$; for $a \leq 39$, we replace $S_{1}^{4}$ and $S_{2}^{4}$ with $S_{1}^{6}$ and $S_{2}^{6}$, and for $11 \leq a \leq 16$, we invoke the full power of $S_{1}^{7}$ and $S_{2}^{7}$. Treating the entire range $11 \leq a \leq 215$ took less than 1.8 seconds using PARI/GP.-For $a \leq 10$, we calculate $\eta_{1}^{(1)}$ to higher precision using a numeric Newton iteration, and compute $\beta$ from the result with adequate safety margins on either side.

The proof of the Theorem is now completed by combining the two propositions with the nonexistence of good diophantine approximations as established in this section.

\section{REFERENCES}

[1] H. Cohen: A Course In Computational Algebraic Number Theory. Springer-Verlag, Berlin et al. (Grad. Texts in Math. 138), 1993. MR 94i:11105

[2] V. Ennola: Cubic number fields with exceptional units. In Computational Number Theory, Proc. of the Colloquium held at Debrecen, September 1989. Ed. A. Pethő, M. E. Pohst, H. C. Williams, and H. G. Zimmer. Walter de Gruyter, 103-138, 1991. MR 93e:11131

[3] G.H. Hardy and E. M. Wright: An introduction to the theory of numbers. Fifth edition. Oxford University Press, 1979. MR 81i:10002

[4] M. Laurent, M. Mignotte and Yu. Nesterenko: Formes linéaires en deux logarithmes et déterminants d'interpolation. J. Number Theory, Vol. 55, 285-321, 1995. MR 96h:11073

[5] H. W. Lenstra Jr.: Euclidean number fields of large degree. Invent. Math., Vol. 38, 237-254, 1977. MR 55:2836

[6] A. Leutbecher and G. Niklasch.: On cliques of exceptional units and Lenstra's construction of euclidean fields. In Number Theory, Proc Jour. Arith., Ulm 1987. Ed. H. P. Schlickewei and E. Wirsing, 150-178. Springer-Verlag, LNM 1380, 1989. MR 90i:11123

[7] M. Mignotte: Verification of a conjecture of E. Thomas. J. Number Theory, Vol. 44, 172-177, 1993. MR 94m:11035

[8] M. Mignotte, A. Pethő and R. Ralf: Complete solutions of a family of quartic Thue and index form equations. Math. Comp., Vol. 65, 341-354, 1996. MR 96d:11034

[9] T. Nagell: Sur une propriété des unités d'un corps algébrique. Arkiv f. Matem., Vol. 5, 343356, 1964. MR 32:7542

: Sur les unités dans les corps biquadratiques primitifs du premier rang. loc. cit. Vol. 7 , 359-394, 1968. MR 39:5511 
_: Quelques problèmes relatifs aux unités algébriques. loc. cit. Vol. 8, 115-127, 1969. MR 42:3053 42:3064

: Sur un type particulier d'unités algébriques. loc. cit. Vol. 8, 163-184, 1969. MR

[10] G. Niklasch: Family portraits of exceptional units. Preprint MPI 95-117, Max-Planck-Inst. f. Math., Bonn, 1995.

[11] A. Pethö: Complete solutions to families of quartic Thue equations. Math. Comp., Vol. 57, 777-798, 1991. MR 92e:11023

[12] N. P. Smart: The solution of triangularly connected decomposable form equations. Math. Comp., Vol. 64, 819-840, 1995. MR 95f:11110

[13] N. P. Smart: Solving Discriminant Form Equations via Unit Equations. J. Symbolic Computation, Vol. 21, 367-374, 1996. MR 97g:11029

[14] E. Thomas: Complete solutions to a family of cubic diophantine equations. J. Number Theory, Vol. 34, 235-250, 1990. MR 91b:11027

[15] N. Tzanakis and B.M.M. de Weger: On the practical solution of the Thue equation. J. Number Theory, Vol. 31, 99-132, 1989. MR 90c: 11018

[16] N. Tzanakis and B.M.M. de Weger: How to explicitly solve a Thue-Mahler equation. Comp. Math., Vol. 84, 223-288, 1992. MR 93k:11025

Zentrum Mathematik Der TU / SCM, Technische Universität München, D-80290 MünChen, Germany

E-mail address: nikl@mathematik.tu-muenchen.de

Institute of Mathematics and Statistics, University of Kent at Canterbury, CanTERBURY, KENT, ENGLAND

E-mail address: N.P.Smart@ukc.ac.uk

Current address: Hewlett-Packard Laboratories, Fitton Road, Stoke Gifford, Bristol, BS12 6QZ, United Kingdom

E-mail address: nsma@hplb.hpl.hp.com 